\title{
XIAP underlies apoptosis resistance of renal cell carcinoma cells
}

\author{
WEN ZHENG YANG ${ }^{1}$, HAIJIANG ZHOU ${ }^{2}$ and YONG YAN ${ }^{3}$ \\ ${ }^{1}$ Department of Anesthesiology, Beijing Shijitan Hospital; ${ }^{2}$ Department of Emergency Medicine, Beijing Chao-Yang \\ Hospital; ${ }^{3}$ Department of Urology, Beijing Shijitan Hospital, Capital Medical University, Beijing 100038, P.R. China
}

Received February 12, 2017; Accepted September 6, 2017

DOI: $10.3892 / \mathrm{mmr} .2017 .7925$

\begin{abstract}
X-linked inhibitor of apoptosis (XIAP), a key member of the inhibitors of apoptosis protein family, can inhibit apoptosis by directly binding to the initiator caspase-9, -3 and -7 , thereby promoting tumor cell survival during tumor progression. In the present study, XIAP basal expression levels were investigated and its contribution to the resistance to apoptosis was evaluated, in the RCC cell lines exposed to apoptosis-inducing drugs. This was investigated by histological methods and western blot analysis. Using RNA interference, elimination of XIAP in Caki-1 cells was also studied, and its contribution to the sensitivity to apoptosis induced through the intrinsic pathway was observed. Differences in XIAP expression were detected between ClearCa- 2 and ClearCa- 6 cell lines. ClearCa- 6 cells with lower expression of XIAP were more sensitive to apoptosis-inducing drugs, compared with ClearCa-2 cells. However, the levels of XIAP expression in both cell lines were stable during apoptosis. Furthermore, a Caki-1 cell line with no XIAP expression was used, and was demonstrated to be more sensitive to the apoptosis induced by the mitochondrial pathway. These results suggested that downregulation of XIAP expression could enhance the sensitivity of RCC cells to apoptosis, and the basal expression of XIAP during apoptosis is stable. This may provide novel insight for targeted gene therapy against XIAP, in the clinic.
\end{abstract}

\section{Introduction}

Renal cell carcinoma (RCC) has obvious resistance to apoptosis induced by chemical and immunological preparations and radiation, and this is why the non-surgical treatment for RCC is unlikely to be effective $(1,2)$. However, the mechanism of RCC in apoptosis resistance is still unclear. Recently, molecular targeting therapy employing multi-kinase inhibitors (MKIs) such as tyrosine kinase inhibitors has raised hope for

Correspondence to: Professor Yong Yan, Department of Urology, Beijing Shijitan Hospital, Capital Medical University, 10 YangFangDian Tieyi Road, Haidian, Beijing 100038, P.R. China E-mail: yanyongcq@163.com

Key words: renal cell carcinoma, apoptosis, X-linked inhibitor of apoptosis, RNA interference patients with advanced RCC; sorafenib and sunitinib $(3,4)$ are becoming first-line treatments for metastatic RCC. However, if the patients develop resistance to MKIs, they rapidly succumb to the disease. It is now of utmost importance to find another way to control cancer progression. Manipulation of the apoptotic mechanisms is one promising approach (5).

Different apoptotic signals such as chemicals can induce cell apoptosis in two ways, one of which is death receptor pathway (extrinsic), the other one is the mitochondrial pathway (intrinsic). Cluster of differentiation (CD)95 antibodies (CH11) are Fas ligands which can induce apoptosis through death receptor pathway $(6,7)$. Topotecan and Etoposide (8) are anti-cancer drugs which can inhibit DNA topoisomerase I and II and induce apoptosis through the mitochondrial pathway $(9,10)$. As an anti-cancer and anti-HIV drug, caffeic acid phenethyl ester (CAPE) is a nuclear factor $(\mathrm{NF})-\kappa \mathrm{B}$ inhibitor. As $\mathrm{NF}-\kappa \mathrm{B}$ targets, the expression of inhibitor of apoptosis proteins (IAPs) can be promoted by $\mathrm{NF}-\kappa \mathrm{B}$, so CAPE induces apoptosis through suppression of IAPs expression (11). However, regardless of which apoptosis pathway is active, the glutathione protease family (caspase) such as caspase-3, -7 and-9 must be activated, causing a chain reaction leading to apoptosis (12). More importantly, caspase- 3 and -7 activation is the key to apoptosis; therefore, once they are activated, apoptosis can be carried out normally. IAPs directly bind to caspases and inhibit their activation, serving a vital role in the regulation of cell apoptosis. Caspase inhibitor XIAP is the most effective among the IAPs family. It can restrain apoptosis by suppressing the apoptosis initiation factor caspase- 9 and effector caspase-3 and -7 (13). Therefore, XIAP expression levels may directly determine the sensitivity of tumor cells to apoptosis.

RNA interference (RNAi) can efficiently and specifically inhibit homologous gene expression (14-17). It interferes with the homologous sequences and gene expression at the transcriptional level, and causes the specific degradation of homologous mRNA, corresponding to silencing gene expression. RNAi technologies can effectively inhibit XIAP expression $(18,19)$. It may reduce the experimental error, and improve the experimental accuracy and credibility.

The present study induced apoptosis in different RCC cell lines, which exhibit varying expression levels of XIAP, through death receptor, mitochondrial and $\mathrm{NF}-\kappa \mathrm{B}$ signaling pathways. Furthermore, RNAi was used to reduce the expression of XIAP in XIAP over-expressing RCC cells in order to study its role in apoptosis, and to investigate the mechanism 
of RCC cells in apoptosis resistance. This way, its potential application value in tumor gene therapy was investigated.

\section{Materials and methods}

Cell culture. The established RCC cell lines ClearCa-2 and ClearCa-6 were obtained from Heinrich-Heine University (Dusseldorf, Germany). The Cakil cell line was purchased from China Infrastructure of Cell Line Resources (Beijing, China), and cultured as described previously (20). CH11 (CD95-specific CH11 antibody) was purchased from Immunotech; Beckman Coulter, Inc. (Brea, CA, USA); Etoposide was from Sigma-Aldrich; Merck KGaA (Darmstadt, Germany), CAPE and Topotecan hydrochloride was from Merck KGaA.

Western blotting. Western blotting was performed on the cell lines, as described previously (20). A horseradish peroxidase-labelled secondary antibody (cat. no. $0101 ; 1: 10,000 ; 37^{\circ} \mathrm{C}$ for $40 \mathrm{~min}$ ) was used and blots were visualized using a Super Signal West Pico Substrate (Pierce; Thermo Fisher Scientific, Inc.) according to the manufacturer's protocol. $\beta$-actin was used as a loading control. The images were analyzed using UN-SCAN-Itgel Automated Digitizing System software (version 5.1 for Windows; Silk Scientific Inc., Orem, UT, USA). The following antibodies were used: Anti-XIAP (cat. no. 2042; $1: 10,000 ; 37^{\circ} \mathrm{C}$ for $40 \mathrm{~min}$ ) from Sigma-Aldrich; Merck KGaA; polyclonal anti-inhibitor of apoptosis 1 (c-IAP1; cat. no. 4952; $1: 10,000 ; 37^{\circ} \mathrm{C}$ for $40 \mathrm{~min}$ ), anti-survivin (cat. no. 2802 ; $1: 10,000 ; 37^{\circ} \mathrm{C}$ for $40 \mathrm{~min}$ ) from Cell Signaling Technology Inc. (Danvers, MA, USA); anti-c-IAP2 (clone F30-2285; 1:10,000; $37^{\circ} \mathrm{C}$ for $40 \mathrm{~min}$ ) from BD Biosciences (Franklin Lakes, NJ, USA) and anti- $\beta$-actin (cat. no. $8227 ; 1: 20,000 ; 37^{\circ} \mathrm{C}$ for $40 \mathrm{~min}$ ) from Abcam (Cambridge, MA, USA).

RNAi. The XIAP-targeting short hairpin RNA vector was generated through literature review (21). The target sequence is 50-AGGTGAAGGTGATAAAGTA-30 (22) Transfection was carried out using Lipofectamine 2000 transfection reagent (Gibco; Thermo Fisher Scientific, Inc.) and a BLOCK-iT ${ }^{\mathrm{TM}}$ U6 RNAi Entry Vector kit (Kang Wei Technology, China; http://www.cwbiotech.com). For generation of stable transfectant clones, the transfected cells were selected with G418 for 3-4 weeks. A total of three selected clones were screened for XIAP expression (clone nos 1-3), and clone no. 2 was randomly selected for further experiments. G418-resistant mock transfectants were also isolated, produced by transfection of the plasmid without XIAP-targeting sequence.

Measurement of cell viability and cell apoptosis. CH11 (cat. no. $49516 ; 37^{\circ} \mathrm{C}$ for $24 \mathrm{~h}$ ) from Abcam (Cambridge, MA, USA), Topotecan (cat. no. d1916; $37^{\circ} \mathrm{C}$ for $24 \mathrm{~h}$ ) from Baomanbio (Shanghai, China), CAPE (cat. no. 211200; $37^{\circ} \mathrm{C}$ for $24 \mathrm{~h}$ ) from Calbiochem; Merck KGaA (Darmstadt, Germany), and Etoposide (cat. no. $341205 ; 37^{\circ} \mathrm{C}$ for $24 \mathrm{~h}$ ) from Calbiochem were used to induce apoptosis, and cell viability was detected by counting cells under the optical microscope. Trypan blue (cat. no. GD-jk $1413 ; 37^{\circ} \mathrm{C}$ for $1 \mathrm{~min}$ ) from Guduo (Shanghai, China) was used to detect viable cells or cell death according to the manufacturer's protocol. In additional

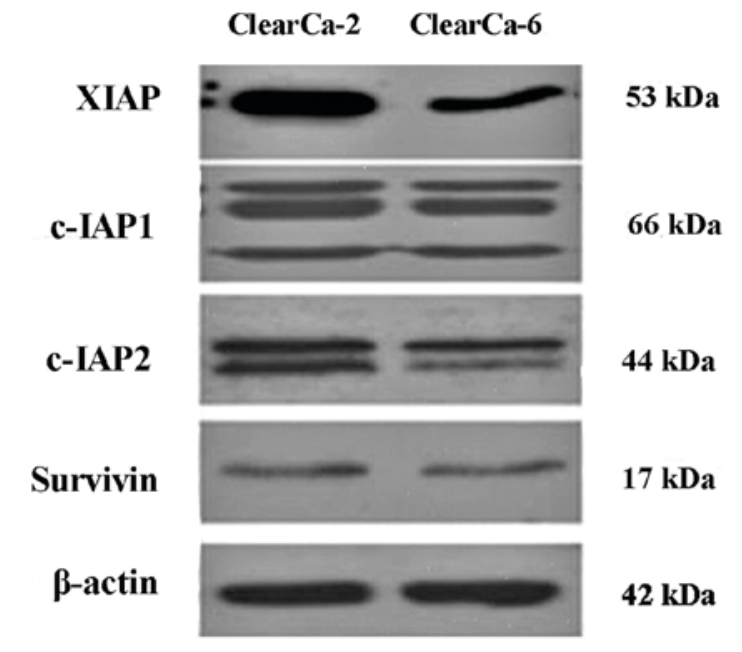

Figure 1. Protein expression of IAP1 and -2, XAP and survivin, in ClearCa-2 and ClearCa- 6 cell lines, as assessed by western blot analysis. XIAP, X-linked inhibitor of apoptosis; c-IAP, inhibitor of apoptosis protein.

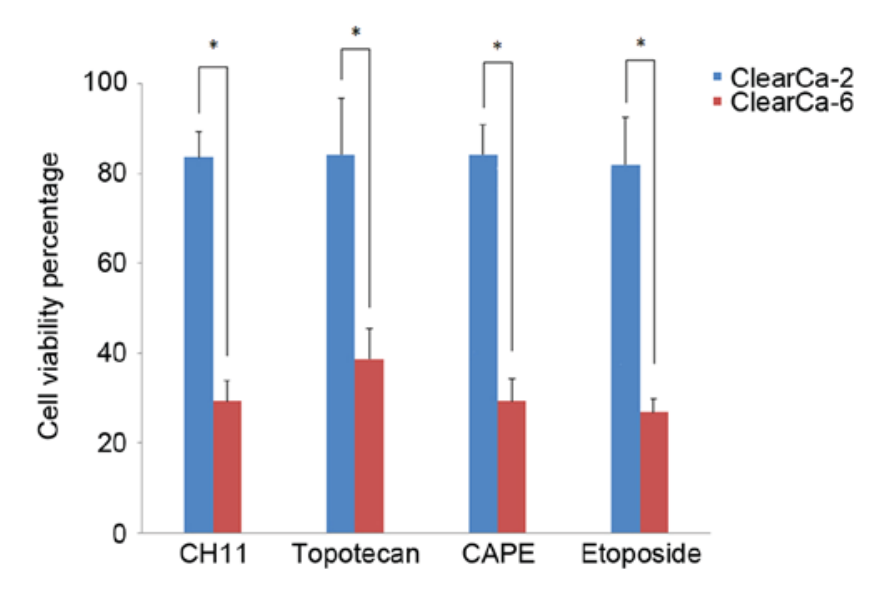

Figure 2. Cell viability percentage of ClearCa-2 and ClearCa- 6 cell lines following $\mathrm{CH} 11$, Topotecan, CAPE and Etoposide treatment. Data are expressed as the mean \pm standard deviation. ${ }^{*} \mathrm{P}<0.05$ ClearCa-2 vs. ClearCa-6. CAPE, caffeic acid phenethyl ester; CH11, CD95-specific antibody.

experiments, in order to improve the experiment efficiency, an MTT kit was used (Gibco; Thermo Fisher Scientific, Inc.) and flow cytometry (BD Biosciences) were used to detect early apoptosis according the manufacturer's protocol.

Statistical analysis. SPSS software version 13.0 was used (SPSS, Inc., Chicago, IL, USA). All data are expressed as the mean \pm standard deviation of three independent experimental replicates. Statistical analysis was performed using a one-way analysis of variance followed by the Least Significant difference post hoc test or Student's t-test. $\mathrm{P}<0.05$ was considered to indicate a statistically significant difference.

\section{Results}

Basal expression of XIAP is significantly different between ClearCa-2 and ClearCa- 6 cell lines. The basal protein expression of XIAP, cIAP-1, cIAP-2 and survivin, which are the main members of the IAPs family, were analyzed by western blot analysis. It was demonstrated that there were no 


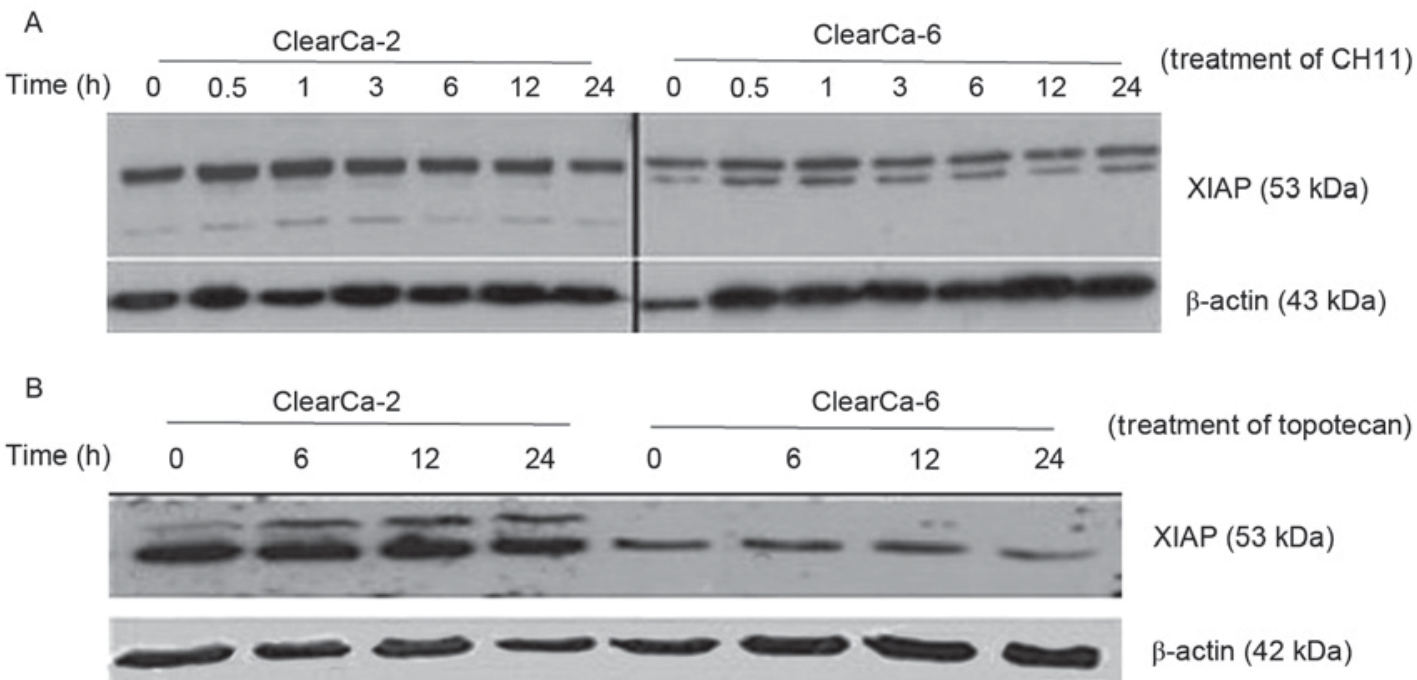

Figure 3. XIAP protein expression following Topotecan and CH11 treatment, as assessed by western blot analysis. Representative western blot images of Clear-Ca-2 and -6 cells following (A) CH11 and (B) Topotecan treatment. XIAP, X-linked inhibitor of apoptosis; CH11, CD95-specific antibody.

differences between cIAP-1 and survivin expression levels in both cell lines, and cIAP-2 had a slight difference, but the expression XIAP between the two cell lines was very different. The expression of XIAP in ClearCa-2 was much higher than ClearCa-6 (Fig. 1).

ClearCa-2 cells with high expression XIAP are resistant to apoptosis; however, ClearCa-6 cells with low-expression XIAP are sensitive to apoptosis. Because of the complicated mechanism of apoptosis, four different drugs which could induce cell apoptosis through different pathways were used. The results demonstrated that ClearCa- 2 cells with higher expression of XIAP were resistant to apoptosis induced by all four drugs (Fig. 2). Following drug treatment for $24 \mathrm{~h}$, the cell viabilities (compared with the control group) were $83.54 \%$ for CH11, 84.07\% for Topotecan, $84.04 \%$ for CAPE, and $81.85 \%$ for Etoposide. However, ClearCa- 6 cells with lower expression of XIAP were sensitive to apoptosis induced by all four drugs (Fig. 2). This difference in the expression levels between the two cell lines was statistically significant $(\mathrm{P}<0.05)$.

Different apoptotic signals did not impact on the expression of XIAP during apoptosis. ClearCa- 2 and -6 cell lines demonstrated a different sensitivity to apoptosis, because of different basal protein expression levels of XIAP. However, whether these levels would change during apoptosis induced by different drug sat different time points or not was investigated. Following $24 \mathrm{~h}$ treatment with CH11 (Fig. 3A) and Topotecan (Fig. 3B), both cell lines had no change in XIAP expression but XIAP expression levels were higher in ClearCa-2 cells compared with the ClearCa- 6 cells.

RNAi technology inhibits the expression of XIAP in the Caki-1 cell line. To further determine the role XIAP serves in RCC cell apoptosis, another XIAP-high-expression RCC cell line was studied (Caki-1), by inhibiting XIAP expression through RNAi. A total of three stable transfection cell lines were used (clone nos. 1-3), and western blot analysis was performed to detect XIAP expression in them. Expression

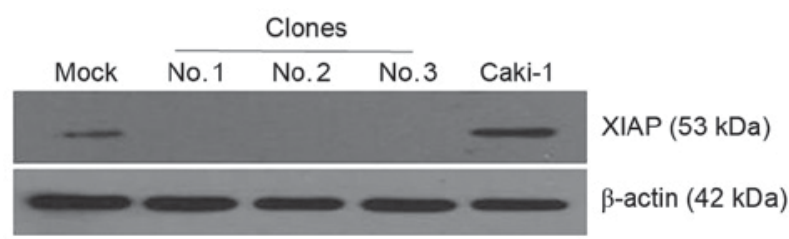

Figure 4. XIAP expression in RNA interference clone cell lines, as assessed by western blot analysis. XIAP, X-linked inhibitor of apoptosis.

levels of XIAP were effectively silenced in all three clone cell lines, but in the parental and the mock group, the expression of XIAP was normal (Fig. 4). Therefore, clone no. 2 was selected in further experiments, and was termed as XIAP no-expression Caki-1 cells.

Sensitivity of Caki-1 cells not expressing XIAP to Etoposide-induced apoptosis is greatly enhanced. It was demonstrated that RCC cells were more sensitive to Etoposide-induced apoptosis than with the other drugs; therefore, XIAP no-expression Caki-1 cells, Caki-1 cells and Mock cells (transfected by plasmid without XIAP interference gene) were treated with Etoposide for $24 \mathrm{~h}$, and the cell death percentage was measured by MTT. It was demonstrated that the cell death percentage of XIAP no-expression Caki-1 cells was much higher than the Caki-1 and Mock cells, $(\mathrm{P}<0.05$; Fig. 5). Between Caki-1 and mock cells, there was no statistical significance in the cell death percentage $(\mathrm{P}=0.519$; Fig. 5A). It was also observed that following the treatment of Etoposide, the early apoptosis rate in XIAP no-expression Caki-1 cells was much higher than in the Caki-1 cells. At $0,0.5,1,3,6$, 12 and $24 \mathrm{~h}$, the early apoptosis rates of XIAP no-expression Caki-1 cells were 1.23, 11.7, 13.87, 22.07, 29.14, 31.81 and $43.21 \%$, respectively. However, the early apoptosis rates of Caki-1 cells were 1.16, 1.48, 2.62, 4.61, 4.61, 3.26, and 5.20\%, respectively (Fig. 5B).

Expression of XIAP is stable during apoptosis induced by Etoposide. In order to observe the level of expression of XIAP 

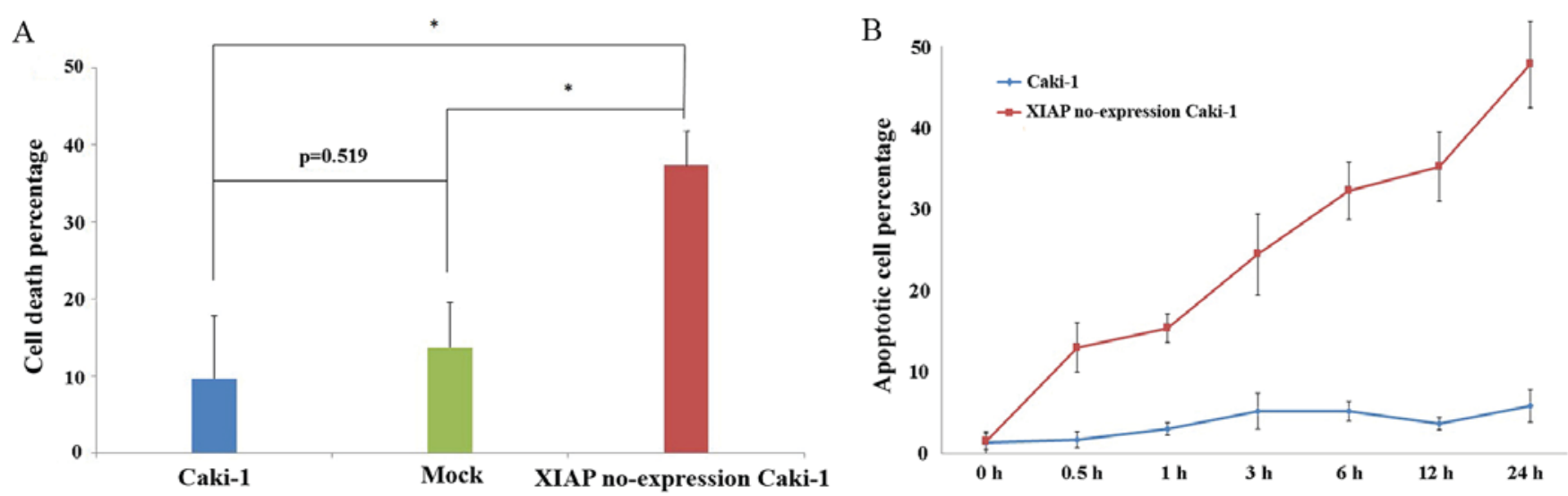

Figure 5. (A) Cell death percentage of Caki-1, mock and RNA interference clone no.2 cells detected by MTT. (B) Time course measurement of the apoptotic cell percentage of Caki-1 and XIAP no-expression Caki-1 cells following Etoposide treatment. As assessed by flow cytometry. Data are expressed as the mean \pm standard deviation. "P<0.05 vs. XIAP no expression Caki cells. XIAP, X-linked inhibitor of apoptosis.
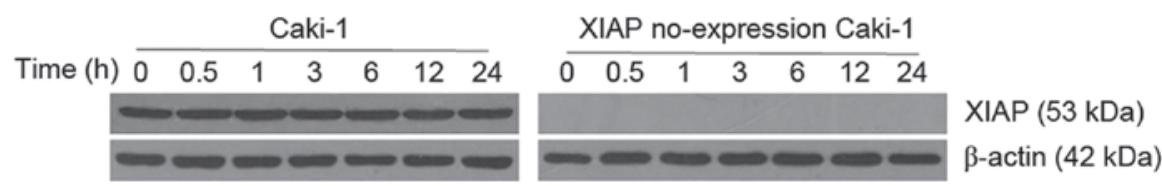

Figure 6. XIAP protein expression levels following Etoposide treatment, as assessed by western blot analysis. XIAP, X-linked inhibitor of apoptosis.

in the progress of apoptosis, the expression of XIAP in Caki-1 and XIAP no-expression Caki-1 cells was detected by western blot analysis, at different time points during apoptosis induced by Etoposide. It was observed that there was no change in the XIAP protein expression levels, as was also observed in the ClearCa-2 an ClearCa-6 cell lines (Fig. 6).

\section{Discussion}

In recent years, many apoptosis controlling genes have been reported (23-25). XIAP, an important member of the anti-apoptotic IAPs family, has a strong inhibitory effect on caspase-3, -7, and -9 and it can also inhibit pro-caspase-3. It can also affect signaling pathways, such as c-Jun N-terminal kinase where, it is thought to serve a key role in the regulation of apoptosis (26-28). Many malignant tumors, such as breast, ovarian, lung, pancreatic and prostate cancer, express high levels of XIAP (29-34). Some reports have mentioned that increasing expression levels of XIAP through transfection can enhance their resistance to apoptosis induced by the death receptors or gamma-rays. Conversely, reducing the expression of XIAP increases the sensitivity of cancer cells to apoptosis $(35,36)$. A previous study illustrated that XIAP is expressed in different histologic types of RCC, and further confirmed the universality of XIAP expression in human cancer (37). Notably, the expression of XIAP in RCC is increased, from early to late cancer stages, at both mRNA and protein levels (37). This indicated that tumor progression coincided with higher expression of XIAP. Similar reports have been presented regarding non-small cell lung cancer and acute myeloid leukemia (38). High expression levels of XIAP may reduce the sensitivity of RCC cells to apoptosis, and provide favorable conditions for tumor cell survival and development.
The present study demonstrated that the expression levels of XIAP have important effects in RCC cells apoptosis. The ClearCa-2 and ClearCa-6 RCC cell lines have different basal protein expression levels of XIAP. Apoptosis may be induced through extrinsic or intrinsic signaling pathways, and ultimately activate caspases leading to apoptosis. A total of four drugs were chosen that function through the extrinsic death receptor pathway $(\mathrm{CH} 11)$, the intrinsic mitochondrial pathway (Etoposide and Topotecan) and the nuclear factor (NF)- $\kappa \mathrm{B}$ inhibiting IAPs (CAPE) induced apoptosis. It was demonstrated that the ClearCa- 6 cell line was sensitive to apoptosis, whereas ClearCa-2 cells were not, and the intrinsic mitochondrial pathway was the strongest in inducing apoptosis. Furthermore, another RCC cell line was employed, termed Caki-1, which has also high expression of XIAP, to verify our conclusion that XIAP may have important effects in RCC cell apoptosis (22). The protein expression of XIAP in theCaki-1 cells was eliminated through RNAi, and it was confirmed that the sensitivity of RCC cells to apoptosis was increased with decreased expression of XIAP.

Previous studies have reported that the expression of XIAP increases following exposure of tumor cells to tumor necrosis factor-related apoptosis-inducing ligand or gamma-rays (39); however, other reports demonstrated the opposite result $(40,41)$. In the present study, CH11 and Topotecan induced apoptosis, but had no effect in the expression of XIAP in either ClearCa-2 or ClearCa- 6 cell lines. The expression of XIAP in both cell lines was stable during apoptosis, although ClearCa-2 cells expressed much higher XIAP than the ClearCa- 6 cells. To verify this result, the expression of XIAP was investigated in Caki-1 cells and no-expression XIAP Caki-1 cells during apoptosis induced by Etoposide, and the same phenomenon was observed. Therefore, it is evident that that the role of $\mathrm{XIAP}$ in apoptosis is very important. 
RNAican effectively suppress protein expression, with a range in the inhibition rate (14-17). Regarding XIAP, Wang et al (18) has reported that RNAi could reduce the expression of XIAP by $56.2 \%$ in laryngeal carcinoma cells. Cao et al (19) has reported that RNAi could reduce its expression by $79.86 \%$ in pancreatic carcinoma cells. Regarding the RCC cells, Bilim et al (22) used RNAi to decrease XIAP by $85.3 \%$, in Caki- 1 cells. In the present study, the interference plasmid transfected Caki-1 cells and it completely inhibited XIAP expression by $100 \%$. To the best of our knowledge, no previous study has ever reported such an effect. This study provides novel insights on XIAP and gene therapy in clinical practice.

In conclusion, there are so many factors inducing apoptosis, and the present study only studied the effect of XIAP on apoptosis. There was a difference in the basal expression of XIAP in two RCC cell lines, and those with higher expression of XIAP resisted apoptosis. At the same time, reducing the expression of XIAP enhanced the sensitivity to apoptosis. However, the underlying mechanism(s) of this phenomenon need to be elucidated further.

\section{Acknowledgements}

The present study was a part of 'Implication of Smac/DIABLO to Resistance to Apoptosis in Renal Cell Carcinoma Cell Lines' (grant no. KM201310025017), the Beijing Municipal Commission of Education, Science and Technology Plan, the 'Comparison of apoptosis - sensitizing mechanisms of XIAP differentially expressed renal cell carcinoma cells' (grant no. 81441073) and the National Natural Science Foundation of China.

\section{References}

1. Yagoda A, Abi-Rached B and Petrylak D: Chemotherapy for advanced renal-cell carcinoma: 1983-1993. Semin Oncol. 22: 42-60, 1995.

2. Spencer WF, Linehan WM, Walther MM, Haas GP, Lotze MT, Topalian SL, Yang JC, Merino MJ, Lange JR, Pockaj BA, et al: Immunotherapy with interleukin-2 and alpha-interferon in patients with metastatic renal cell cancer with in situ primary cancers: A pilot study. J Urol 147: 24-30, 1992.

3. Ljungberg B, Hanbury DC, Kuczyk MA, Merseburger AS, Mulders PF, Patard JJ and Sinescu IC: European Association of Urology Guideline Group for renal cell carcinoma. Renal cell carcinoma guideline. Eur Urol 51: 1502-1510, 2007.

4. Motzer RJ and Bukowski RM: Targeted therapy for metastatic renal cell carcinoma. J Clin Oncol 24: 5601-5608, 2006.

5. Tamm I, Kornblau SM, Segall H, Krajewski S, Welsh K, Kitada S Scudiero DA, Tudor G, Qui YH, Monks A, et al: Expression and prognostic significance of IAP-family genes in human cancers and myeloid leukemias. Clin Cancer Res 6: 1796-1803, 2000

6. Nagata S: Apoptosis by death factor. Cell 88: 355-365, 1997.

7. Watabe M, Hishikawa K, Takayanagi A, Shimizu N and Nakaki T: Caffeic acid phenethyl ester induces apoptosis by inhibition of NFkappaB and activation of Fas in human breast cancer MCF-7 cells. J Biol Chem 279: 6017-6026, 2004.

8. Liang Lu, Dong Li and Fuchu He: Advances in bioinformatics of ubiquitination of protein. Hereditas 35: 17-26, 2013 (In Chinese).

9. Adams JM and Cory S: The Bcl-2 protein family: Arbiters of cell survival. Science 281: 1322-1326, 1998

10. Kothakota S, Azuma T, Reinhard C, Klippel A, Tang J, Chu K, McGarry TJ, Kirschner MW, Koths K, Kwiatkowski DJ and Williams LT: Caspase-3-generated fragment of gelsolin: Effector of morphological change in apoptosis. Science 278: 294-298, 1997.

11. Watabe M, Hishikawa K, Takayanagi A, Shimizu N and Nakaki T: Caffeic acid phenethyl ester induces apoptosis by inhibition of NFkappaB and activation of Fas in human breast cancer MCF-7 cells. J Biol Chem 279: 6017-6026, 2004.
12. Chang HY and Yang X: Proteases for cell suicide: Functions and regulation of caspases. Microbiol Mol Biol Rev 64: 821-846, 2000.

13. Holcik M and Korneluk RG: XIAP, the guardian angel. Nat Rev Mol Cell Biol 2: 550-556, 2001.

14. Yamada T, Horinaka M, Shinnoh M, Yoshioka T, Miki T and Sakai T: A novel HDAC inhibitor OBP-801 and a PI3K inhibitor LY294002 synergistically induce apoptosis via the suppression of survivin and XIAP in renal cell carcinoma. Int J Oncol 43: 1080-1086, 2013

15. Shao SL, Cui TT, Zhao W, Zhang WW, Xie ZL, Wang CH, Jia HS and Liu Q: RNAi-based knockdown of multidrug resistance-associated protein 1 is sufficient to reverse multidrug resistance of human lung cells. Asian Pac J Cancer Prev 15: 10597-105601, 2014.

16. Guo SY, Zhu XD, Ge LY, Qu S, Li L, Su F and Guo Y: RNAi-mediated knockdown of the c-jun gene sensitizes radioresistant human nasopharyngeal carcinoma cell line CNE-2R to radiation. Oncol Rep 33: 1155-1160, 2015.

17. Patrick J. Wightman, George R. Jackson and Katrina M. Dipple: Disruption of glycerol metabolism by RNAi targeting of genes encoding glycerol kinase results in a range of phenotype severity in Drosophila. PLoS One 8: e71664, 2013.

18. Wang R, Li B, Wang X, Lin F, Gao P, Cheng SY and Zhang HZ: Inhibiting XIAP expression by RNAi to inhibit proliferation and enhance radiosensitivity in laryngeal cancer cell line. Auris Nasus Larynx 36: 332-339, 2009.

19. Cao LP, Song JL, Yi XP and Li YX: Double inhibition of NF- $\kappa B$ and XIAP via RNAi enhances the sensitivity of pancreatic cancer cells to gemcitabine. Oncol Rep 29: 1659-1665, 2013.

20. Tomita Y, Bilim V, Kawasaki T, Takahashi K, Okan I, Magnusson KP and Wiman KG: Frequent expression of Bcl-2 in renal-cell carcinomas carrying wild-type p53. Int J Cancer 66: 322-325, 1996

21. Bilim V, Yuuki K, Itoi T, Muto A, Kato T, Nagaoka A, Motoyama T and Tomita Y: Double inhibition of XIAP and Bcl-2 axis is beneficial for retrieving sensitivity of renal cell cancer to apoptosis. Br J Cancer 98: 941-949, 2008.

22. Bilim V, Yuuki K, Itoi T, Muto A, Kato T, Nagaoka A, Motoyama T and Tomita Y: Double inhibition of XIAP and Bcl-2 axis is beneficial for retrieving sensitivity of renal cell cancer to apoptosis. Br J Cancer 98: 941-949, 2008.

23. Sioud M: siRNA and miRNA Gene Silencing: From Bench to Bedside. Humana Press, New York, NY, 2009.

24. Vorburger SA, Pataer A, Swisher SG and Hunt KK: Gene therapy for cancer. Humana Press, Totowa, NJ, 2007.

25. Potten CS, Wilson JW and Booth C: Apoptosis genes. Kluwer Academic, Boston, 1998.

26. Arroyo JA, Li C, Schlabritz-Loutsevitch N, McDonald T, Nathanielsz P and Galan HL: Increased placental XIAP and caspase 3 is associated with increased placental apoptosis in a baboon model of maternal nutrient reduction. Am J Obstet Gynecol 203: 364.e13-8, 2010.

27. Hörnle M, Peters N, Thayaparasingham B, Vörsmann H, Kashkar H and Kulms D: Caspase- 3 cleaves XIAP in a positive feedback loop to sensitize melanoma cells to TRAIL-induced apoptosis. Oncogene 30: 575-587, 2011.

28. Liu Y, Zhou ZG, Zhou B, Wang R, Yan H and Li Y: Downregulation of GRP78 and XIAP is correlated with apoptosis during cerulein-induced acute pancreatitis in rats via regulation of caspase activation. Mol Med Rep. 7: 725-730, 2013.

29. Cheng YJ, Jiang HS, Hsu SL, Lin LC, Wu CL, Ghanta VK and Hsueh CM: XIAP-mediated protection of H460 lung cancer cells against cisplatin. Eur J Pharmacol 627: 75-84, 2010.

30. Danquah M, Duke CB III, Patil R, Miller DD and Mahato RI: Combination therapy of antiandrogen and XIAP inhibitor for treating advanced prostate cancer. Pharm Res 29: 2079-2091, 2012.

31. Nestal de Moraes G, Vasconcelos FC, Delbue D, Mognol GP, Sternberg C, Viola JP and Maia RC: Doxorubicin induces cell death in breast cancer cells regardless of Survivin and XIAP expression levels. Eur J Cell Biol 92: 247-256, 2013.

32. Castells M, Milhas D, Gandy C, Thibault B, Rafii A, Delord JP and Couderc B: Microenvironment mesenchymal cells protect ovarian cancer cell lines from apoptosis by inhibiting XIAP inactivation. Cell Death Dis 4: e887, 2013.

33. Zai HY, Yi XP, Li YX, You XY, Cao LP and Liu H: X-linked inhibitor of apoptosis protein (XIAP) and Survivin suppression on human pancreatic cancer cells Panc-1 proliferation and chemosensitivety. Beijing da xue xue Bao 45: 242-249, 2013. 
34. Ning ZR, Li S, Guo YW and Fang DJ: Expression and clinical significance of Cox-2 and XIAP in malignant tumors of the salivary gland. Shanghai Kou Qiang Yi Xue 23: 317-321, 2014.

35. Spahn A, Blondeau N, Heurteaux C, Dehghani F and Rami A Concomitant transitory up-regulation of X-linked inhibitor of apoptosis protein (XIAP) and the heterogeneous nuclear ribonucleoprotein $\mathrm{C} 1-\mathrm{C} 2$ in surviving cells during neuronal apoptosis. Neurochem Res 33: 1859-1868, 2008.

36. Holt SV, Brookes KE, Dive C and Makin GW: Down-regulation of XIAP by AEG35156 in paediatric tumour cells induces apoptosis and sensitises cells to cytotoxic agents. Oncol Rep 25: 1177-1181, 2011.

37. Yan Y, Mahotka C, Heikaus S, Shibata T, Wethkamp N, Liebmann J, Suschek CV, Guo Y, Gabbert HE, Gerharz CD and Ramp U: Disturbed balance of expression between XIAP and Smac/DIABLO during tumour progression in renal cell carcinomas. Br J Cancer 91: 1349-1357, 2004.

38. Tamm I, Kornblau SM, Segall H, Krajewski S, Welsh K, Kitada S, Scudiero DA, Tudor G, Qui YH, Monks A, et al: Expression and prognostic significance of IAP-family genes in human cancers and myeloid leukemias. Clin Cancer Res 6: 1796-1803, 2000.
39. Ramp U,Caliskan E, Mahotka C, Krieg A, Heikaus S, Gabbert HE and Gerharz CD: Apoptosis induction in renal cell carcinoma by TRAIL and gamma-radiation is impaired by deficient caspase-9 cleavage. Br J Cancer 88: 1800-1807, 2003.

40. Ng CP, Zisman A and Bonavida B: Synergy is achieved by complementation with Apo2L/TRAIL and actinomycin D in Apo2L/TRAIL-mediated apoptosis of prostate cancer cells: Role of XIAP in resistance. Prostate 53: 286-299, 2002.

41. Flanagan L, Sebastia J, Delgado ME, Lennon JC and Rehm M: Dimerization of Smac is crucial for its mitochondrial retention by XIAP subsequent to mitochondrial outer membrane permeabilization. Biochim Biophys Acta 1813: 819-826, 2011.

This work is licensed under a Creative Commons Attribution-NonCommercial-NoDerivatives 4.0 International (CC BY-NC-ND 4.0) License. 